\title{
Pseudogap and precursor superconductivity in underdoped cuprate high temperature superconductors: A far-infrared ellipsometry study
}

A. Dubroka ${ }^{1}$, L. Yu ${ }^{1, a}$, D. Munzar ${ }^{2}$, K.W. Kim ${ }^{1}$, M. Rössle ${ }^{1}$, V.K. Malik ${ }^{1}$, C.T. Lin $^{3}$, B. Keimer ${ }^{3}$, Th. Wolf ${ }^{4}$, and C. Bernhard ${ }^{1, b}$

${ }^{1}$ Department of Physics and Fribourg Center for Nanomaterials, University of Fribourg, Chemin du Musée 3, 1700 Fribourg, Switzerland

2 Institute of Condensed Matter Physics, Faculty of Science, Masaryk University, Kotlárská 2, 61137 Brno, Czech Republic

${ }^{3}$ Max-Planck-Institut für Festkörperforschung, Heisenbergstrasse 1, 70569 Stuttgart, Germany

${ }^{4}$ Karlsruhe Institute of Technology, 76021 Karlsruhe, Germany

Received 14 July 2010 / Received in final form 12 August 2010

Published online 11 October 2010

\begin{abstract}
With the technique of infrared ellipsometry we performed a detailed study of the temperature- and doping dependence of the $c$-axis response of a series of $\mathrm{YBa}_{2} \mathrm{Cu}_{3} \mathrm{O}_{7-\delta}$ single crystals. In particular, we explored the anomalous electronic properties at temperatures above the macroscopic superconducting transition temperature, $T_{\mathrm{c}}$, whose conflicting explanations range from a precursor superconducting state to electronic correlations that compete with superconductivity. We show that the $c$-axis spectra provide evidence that both kinds of correlations are present and that their contributions can be disentangled based on an analysis with a so-called multilayer-model. We find that the onset temperature, $T^{*}$, and the energy scale, $\Delta_{\mathrm{PG}}$, of the competing pseudogap increase rapidly towards the underdoped side whereas they vanish on the overdoped side. In addition, we provide evidence that in a strongly underdoped sample the precursor superconducting correlations develop below an onset temperature, $T^{\text {ons }}$, that is considerably lower than $T^{*}$ but still much higher than $T_{\mathrm{c}}$.
\end{abstract}

\section{Introduction}

One of the most controversially discussed aspects of the cuprate high temperature superconductors (HTSC) concerns their highly anomalous electronic properties in the so-called normal state at temperatures above the macroscopic superconducting transition, $T_{\mathrm{c}}$. The most prominent and controversially discussed feature is the so-called pseudogap phenomenon which gives rise to an incomplete, gap-like depletion of the low-energy electronic states that sets in already well above $T_{\mathrm{c}}[1-4]$ in samples that are underdoped with respect to the dome-shaped phase diagram of $T_{\mathrm{c}}$ versus the hole doping, $p$, per $\mathrm{CuO}_{2}$ plane $[5,6]$. The signatures of this pseudogap have been observed with several experimental techniques, like nuclear magnetic resonance [7], specific

\footnotetext{
a Actual address: National Laboratory for Superconductivity, Institute of Physics and Beijing National Laboratory for Condensed Matter Physics, Chinese Academy of Sciences, PO Box 603, Beijing 100190, Peoples Republic of China

b e-mail: christian.bernhard@unifr.ch
} 
heat [8], angle-resolved photo-emission (ARPES) [9-11], $c$-axis tunneling [12,13], scanning tunnelling microscopy (STM) [15-18], Andreev reflection [19], Nernst-effect [20], dilatometry [21], and also by far infrared (FIR) spectroscopy [2,22-26]. These have established important aspects, like the strong $k$-space anisotropy of the pseudogap which prevails in the so-called antinodal region of the Fermi-surface near the X-point of the Brillouin-zone at $(0, \pi / \mathrm{a})$ [9-11], or the rapid rise of its onset temperature, $T^{*}$, and energy scale, $\Delta_{\mathrm{PG}}$, on the underdoped side $[4,11,27-29]$. However, the origin of the pseudogap remains unknown. Even the central question whether it arises from a precursor superconducting state where the local pairing occurs at much higher temperature than the onset of macroscopic coherence at $T_{\mathrm{c}}$ (intrinsic scenario), or whether it is caused by a different kind of possibly competing electronic correlation (extrinsic scenario) remains debated. Prominent theories for the intrinsic scenario are the phase fluctuation model where the pseudogap corresponds to a superconducting state that is lacking macroscopic phase coherence [30], precursor pairing models where the pair formation occurs at much higher temperature than the condensation [31], and the resonating valence bond theory [32]. The extrinsic models include numerous conventional and exotic spin- and/or charge density wave (SDW, $\mathrm{CDW})$ states [33-35].

Previous studies of the infrared (IR) $c$-axis conductivity, $\tilde{\sigma}_{c}=\sigma_{1 c}+\mathrm{i} \sigma_{2 c}$, of single crystals of underdoped $\mathrm{YBa}_{2} \mathrm{Cu}_{3} \mathrm{O}_{7-\delta}$ (Y-123) revealed several important aspects of the normal state pseudogap. They were the first to show that, besides the so-called spin gap [7], a partial, gaplike suppression also occurs in the low-energy charge excitations [22]. They also demonstrated that the onset temperature, $T^{*}$, of the suppression of $\sigma_{1 c}$ increases rapidly on the underdoped side exceeding ambient temperature at $p \leq 0.12[29,36-38]$. More recent infrared ellipsometry measurements established that the missing low-energy spectral weight due to the pseudogap in $\sigma_{1 c}$ is transferred to higher energy into a broad band that is located just above the gap edge near $2 \Delta_{\mathrm{PG}}[29,38]$. This is unlike the $\mathrm{SC}$ gap at $T<T_{\mathrm{c}}$ where the missing spectral weight is redistributed into a delta function at $\omega=0$ that accounts for the loss-free response of the condensate [39]. This observation provides strong evidence for an extrinsic scenario of a pseudogap below $T^{*}$ that depletes the density of low-energy states that is available for superconductivity once it sets in at $T_{\mathrm{c}}<T^{*}$.

On the other hand, there exists clear experimental evidence that precursor superconducting correlations persists to temperatures well in excess of the macroscopic $T_{\mathrm{c}}$ value of the underdoped cuprate HTSC based on experimental studies of the Nernst effect and magnetization [20,40,41], Andreev reflection [19], dilatometry [21], and a recent analysis of the specific heat data [42]. In this manuscript we discuss the evidence that the $c$-axis response of the bilayer compound Y-123 also contains the signatures of a second kind of electronic transition in the normal state that can be associated with a precursor superconducting state and thus with an intrinsic scenario. The latter develop below an onset temperature, $T^{\text {ons }}$, that is significantly lower than $T^{*}$ but still much higher than $T_{\mathrm{c}}$. We show that the information about the precursor superconducting state is contained in an electronic mode, the so-called transverse plasma mode (TPM), and in the related anomalous temperature dependence of some infrared-active phonons, which have been previously identified $[22,23,36,43]$. It is meanwhile well established that both of these features can be well described with the so-called multilayer model (MLM) [43-48] where the difference between the electronic response of the bilayer region and that of the inter-bilayer region is taken into account and described in terms of the local conductivities, $\sigma_{\mathrm{bl}}$ and $\sigma_{\mathrm{int}}$, respectively. Below we outline how the characteristic frequency- and temperature dependence of $\sigma_{\mathrm{bl}}$ and $\sigma_{\mathrm{int}}$ can be derived with the MLM and can be used to disentangle the properties and, in particular, the onset temperatures of the precursor superconducting state and the competing pseudogap, $T^{\text {ons }}$ and $T^{*}$, respectively.

\section{Experimental details}

$\mathrm{RBa}_{2} \mathrm{Cu}_{3} \mathrm{O}_{7-\delta}$ single crystals $\left(\mathrm{R}=\mathrm{Y}\right.$, Nd) of typical dimension $2 \times 2 \times 0.5-1 \mathrm{~mm}^{3}$ were grown in Y-stabilized zirconium crucibles as described in Refs. [49,50]. The hole doping of the $\mathrm{CuO}_{2}$ planes, $p$, was adjusted via the oxygen content of the $\mathrm{CuO}$ chain layer, $\delta$, 
by annealing in flowing $\mathrm{O}_{2}$ and subsequent rapid quenching in liquid nitrogen. Alternatively, some strongly underdoped samples were obtained by partial substitution of $\mathrm{R}^{3+}$ with $\mathrm{Ca}^{2+}[6,51,52]$. The quoted values of $T_{\mathrm{c}}$ corresponding to the midpoint of the diamagnetic transition were determined by dc magnetization measurements using a vibration sample magnetometer (PPMS from Quantum Design). The $p$ values were obtained either with the empirical relationship, $p=0.16 \pm \sqrt{\left(1-T_{\mathrm{c}} / T_{\mathrm{c}, \max }\right) / 82.6}[5,6]$, from the measured value of the thermoelectric power (TEP) at room temperature $[51,53]$, or from the Ca-content, $x$, according to $p=x / 2$.

The ellipsometric measurements were performed with a home-built ellipsometer attached to a Bruker Fast-Fourier spectrometer below $700 \mathrm{~cm}^{-1}$ at the infrared beamline of the ANKA synchrotron at FZ Karlsruhe, Germany and at $400-4000 \mathrm{~cm}^{-1}$ with a similar lab-based setup [54-56]. The presented $c$-axis polarized spectra are corrected for anisotropy effects (due to the layered structure of the high- $T_{\mathrm{c}}$ cuprates) by using standard numerical procedures $[56,57]$. Accordingly, each spectrum is based on a set of two independent measurements with either the $a b$ - or the $c$-axis in the plane of incidence (as defined by the incoming and reflected light). Ellipsometry measures the complex dielectric function, $\epsilon(\omega)=\epsilon_{1}(\omega)+\mathrm{i} \epsilon_{2}(\omega)$, and the related optical conductivity,

$$
\sigma(\omega)=\frac{\mathrm{i} \omega}{4 \pi}(1-\epsilon(\omega))
$$

without a need for a Kramers-Kronig analysis [56,57]. Furthermore, it is a self-normalizing and thus very accurate and reproducible technique. Thanks to the large probe depth of the IR radiation of the order of micrometers, which ensures the bulk nature of the observed phenomena, and the availability of powerful sum rules, it provides important complementary information with respect to other spectroscopic techniques like angle resolved photoemission (ARPES) or tunneling spectroscopy.

\section{Competing pseudogap in the $c$-axis conductivity}

The electronic background of the $c$-axis conductivity, $\sigma_{1 c}$, of underdoped R-123 single crystals is well known to exhibit a gap like reduction with decreasing temperature that can be associated with the pseudogap phenomenon $[22,23,25,29,38,58]$. From the $c$-axis conductivity spectra one can readily determine the characteristic pseudogap parameters such as the onset temperature, $T^{*}$, and the onset frequency, $\omega_{\mathrm{PG}} \approx 2 \Delta_{\mathrm{PG}}$, where $\Delta_{\mathrm{PG}}$ is the maximal energy of the pseudogap. More recent ellipsometry measurements revealed that the missing spectral weight due to the suppression of $\sigma_{1 c}\left(\omega<\omega_{\mathrm{PG}}\right)$ is transferred to higher energy, i.e. just above the gap edge where it gives rise to a broad absorption band [27,38]. Most importantly, it was found that this broad absorption band is unaffected by the superconducting transition. In other words, this shows that the pseudogap depletes the density of low-energy states that are available for superconductivity below $T_{\mathrm{c}}$, i.e. it competes with superconductivity. In the following, we briefly revisit the analysis of the $c$-axis conductivity spectra which shows that the pseudogap competes with superconductivity [29]. This is shown in Fig. 1 for the case of a moderately underdoped $\mathrm{NdBa}_{2} \mathrm{Cu}_{3} \mathrm{O}_{6.9}$ single crystal with $T_{\mathrm{c}}=85 \mathrm{~K}$ and $T^{*} \approx 220 \mathrm{~K}$. Figure 1 (a) shows the changes of $\sigma_{1 c}(\omega)$ as the pseudogap develops between $240 \mathrm{~K}$ and $90 \mathrm{~K}$. It can be seen that $\sigma_{1 c}(\omega)$ is suppressed below $\omega_{\mathrm{PG}} \approx 700 \mathrm{~cm}^{-1}$ while it is increased above. Figure $1(\mathrm{~b})$ shows the corresponding spectral changes below $T_{\mathrm{c}}$ where the crossing point, below which the conductivity is suppressed, is at $\omega_{\mathrm{SC}} \approx 860 \mathrm{~cm}^{-1}$. Figure $1(\mathrm{c})$ details the temperature dependence of the regular spectral weight below $800 \mathrm{~cm}^{-1} \mathrm{SW}_{0^{+}}^{800}$. It shows how the onset temperature of the pseudogap phenomenon of $T^{*} \approx 220 \mathrm{~K}$ has been determined from the onset of the suppression of $\mathrm{SW}_{0^{+}}^{800}$.

Figure 1(d) highlights that this temperature dependent decrease of the spectral weight below $T^{*}$ is absent for an upper integration limit of $4000 \mathrm{~cm}^{-1}$. In other words, it confirms that the missing low-frequency spectral weight (below $\omega_{\mathrm{PG}}$ ) gets transferred into the broad band just above the gap edge. It should be noted that the spectral shape of these changes in $\sigma_{1 c}$ resembles 


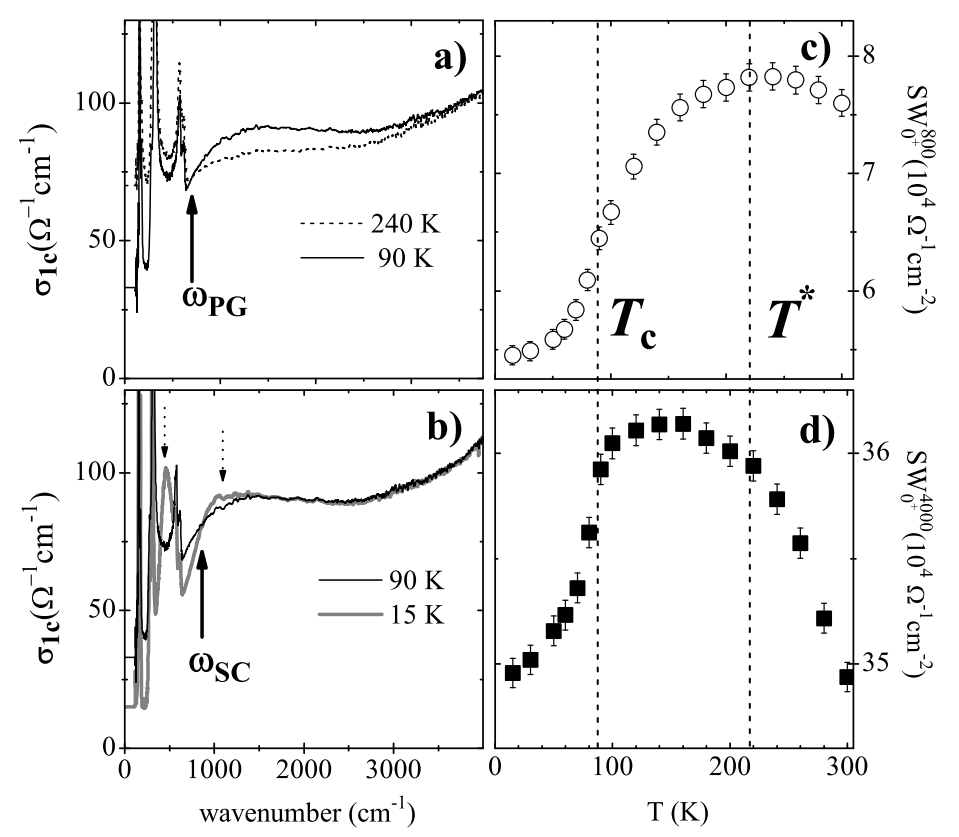

Fig. 1. The real part of the $c$-axis conductivity of underdoped $\mathrm{NdBa}_{2} \mathrm{Cu}_{3} \mathrm{O}_{6.9}$ with $T_{\mathrm{c}}=85 \mathrm{~K}$ (from Ref. [29]). Shown are representative spectra for (a) the pseudogap state and (b) the superconducting state. The gap edges at $\omega_{\mathrm{PG}} \approx 2 \Delta_{\mathrm{PG}}$ and $\omega_{\mathrm{SC}} \approx 2 \Delta_{\mathrm{SC}}$ are marked with the solid arrows. The dotted arrows in (b) show the extra electronic modes which develop below $T_{\mathrm{c}}$ and that are discussed in the next chapter. Shown in (c) and (d) is the temperature dependence of the regular spectral weight below $800 \mathrm{~cm}^{-1}, \mathrm{SW}_{0^{+}}^{800}$, and below $4000 \mathrm{~cm}^{-1}, \mathrm{SW}_{0^{+}}^{4000}$, respectively. The dashed vertical lines mark the onset temperature of the pseudogap, $T^{*}$, and the superconducting transition temperature, $T_{\mathrm{c}}$, respectively.

the one of some BCS-type spin- or charge density wave systems [59]. The spectral weight is also redistributed here from the region below the gap edge to a pairbreaking peak that is located right above the gap edge. However, the temperature dependence of the pseudogap, which does not seem to close as temperature increases but rather fills in with thermally excited states, appears to be clearly different.

Most importantly, we find that the pseudogap-induced broad electronic band above $\omega_{\mathrm{PG}}$ remains unchanged below $T_{\mathrm{c}}$. This is evident from Fig. 1(b) which compares the spectra at $90 \mathrm{~K}$ just above $T_{\mathrm{c}}=85 \mathrm{~K}$ and at $15 \mathrm{~K} \ll T_{\mathrm{c}}$ and shows that they essentially coincide above $1400 \mathrm{~cm}^{-1}$. This behavior is not compatible with the point of view that the pseudogap arises from a precursor superconducting state. In that case one would expect that, as the fluctuations of the phase and/or the amplitude of the order parameter eventually become reduced below $T_{\mathrm{c}}$, parts of the spectral weight of the pseudogap-induced electronic band should be transferred back to low frequency and participate in the superconducting condensate. Instead, our infrared data show that the pseudogap depletes the density of the low-energy states that are available for superconductivity below $T_{\mathrm{c}}$. This suggests that the pseudogap competes with superconductivity for the available low energy electronic states and thus is likely caused by electronic or magnetic correlations that are different from the ones that are at the heart of the superconducting pairing mechanism $[29,38]$.

The evidence for a distinct nature of the pseudogap and the superconducting gap is reinforced by the observation that their magnitudes evolve very differently as a function of the hole doping of the $\mathrm{CuO}_{2}$ planes. In Fig. 2 and 3 we show for a series of weakly overdoped to strongly underdoped Y-123 crystals how these gap magnitudes, $\omega_{\mathrm{PG}}$ and $\omega_{\mathrm{SC}}$, have been determined. The value of $\omega_{\mathrm{PG}}$ has been obtained from the crossing point of the conductivity spectra at $T^{*}$ and $T_{\mathrm{c}}$ and the value of $\omega_{\mathrm{SC}}$ from the crossing point of the spectra at $T_{\mathrm{c}}$ and $T \ll T_{\mathrm{c}}$. We note that the relationship $\omega_{\mathrm{PG}} \approx 2 \Delta_{\mathrm{PG}}$ can be justified with model calculations as presented in 


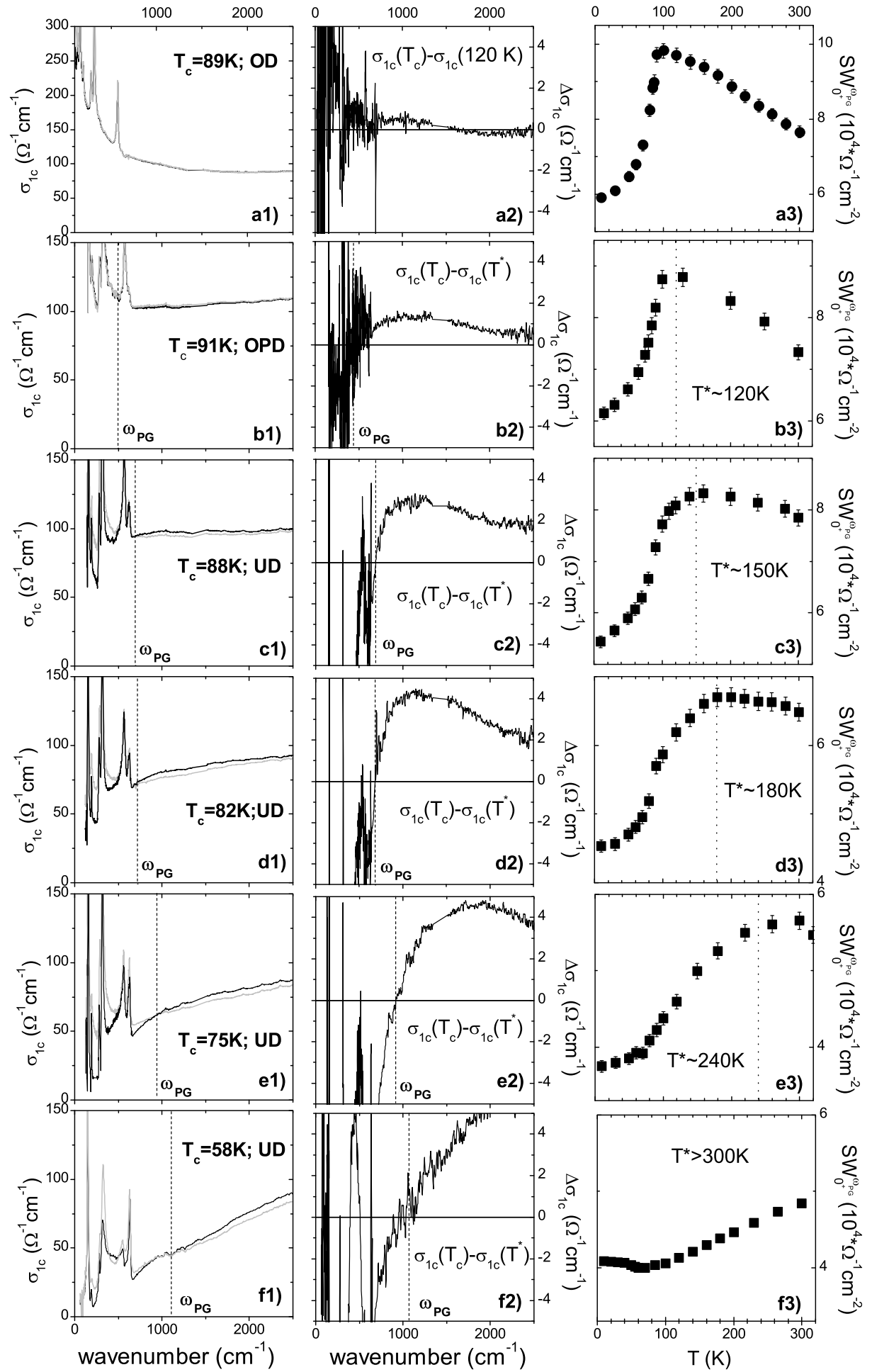

Fig. 2. (a1)-(f1) The real part of the $c$-axis conductivity spectra at $T_{\mathrm{c}}$ (black line) and $T^{*}$ (gray line) for a series of slightly overdoped (OD) to strongly underdoped (UV) Y-123 crystals. The crossing point as shown by the dotted line marks the edge of the pseudogap, $\omega_{\mathrm{PG}}$. Note that no pseudogap is observed for the OD sample. (a2)-(f2) Corresponding difference spectra. (a3)-(f3) Temperature dependence of the low-frequency spectral weight up to $\omega_{\mathrm{PG}}$ from which the onset temperature of the pseudogap, $T^{*}$, has been determined. 


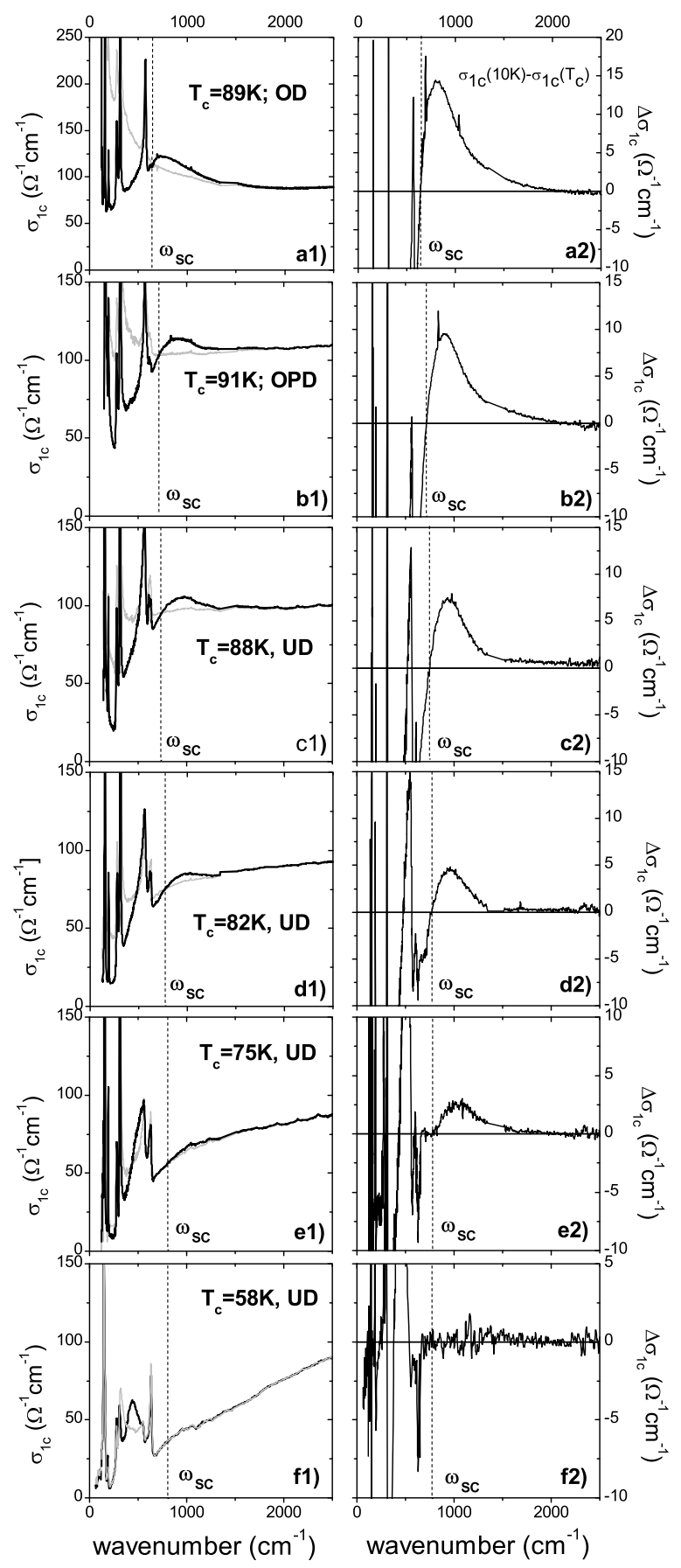

Fig. 3. (a1)-(f1) Spectra of the real part of the conductivity at $T_{\mathrm{c}}$ (gray lines) and $T \ll T_{\mathrm{c}}$ (black lines) for a series of weakly overdoped to strongly underdoped Y-123 single crystals. (a2)-(f2) The corresponding difference spectra detail the SC-induced changes. The dotted lines mark the position of the superconducting gap edge, $\omega_{\mathrm{SC}}$.

Ref. [25] by using fits of the quasiparticle spectral functions of Ref. [60]. The justification of the relation $\omega_{\mathrm{SC}} \approx 2 \Delta_{\mathrm{SC}}$ involves the mode around $1000 \mathrm{~cm}^{-1}$ that occurs right above $\omega_{\mathrm{SC}}$ in the spectra at $T<T_{\mathrm{c}}$ and likely corresponds to an interband (bonding-antibonding) pair-breaking peak whose coherence factor decreases with decreasing bilayer coupling [61,62]. This has been 


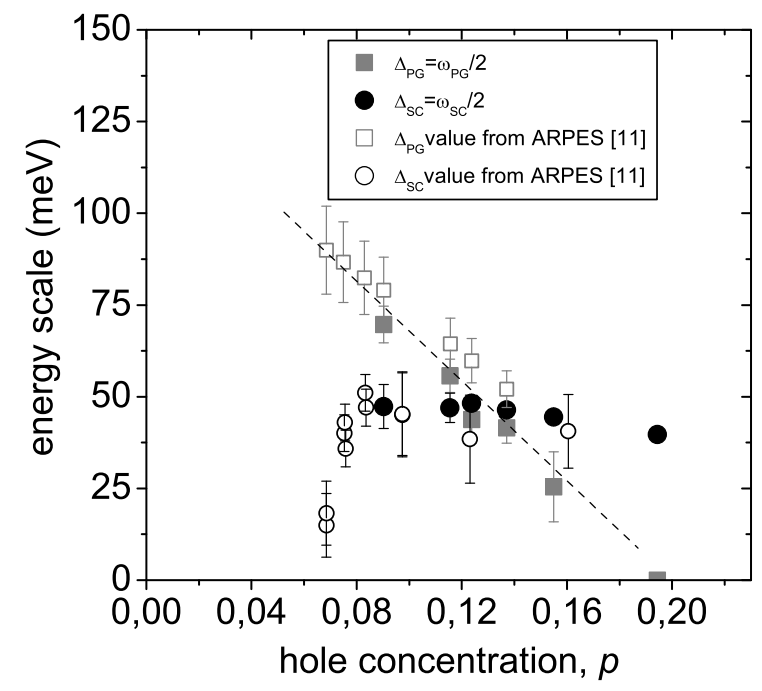

Fig. 4. Doping dependence of the energy scales $\Delta_{\mathrm{PG}} \approx \hbar \omega_{\mathrm{PG}} / 2$ (gray solid squares) and $\Delta_{\mathrm{SC}} \approx \hbar \omega_{\mathrm{SC}} / 2$ (black solid circles) as deduced from Figs. 2 and 3 . The corresponding values for $\Delta_{\mathrm{PG}}$ (gray open squares) and $\Delta_{\mathrm{SC}}$ (black open circles) as reported from high-resolution ARPES measurements on underdoped $\mathrm{Bi}-2212$ single crystals [11]. Here the value of $\Delta_{\mathrm{PG}}$ was obtained from the data near the anti-nodal region in $k$-space and $\Delta_{\mathrm{SC}}$ from the extrapolation of the data near the nodal region.

established in Refs. [29]. According to this interpretation, the pairbreaking mode is located just a few tens of meV above $2 \omega_{\mathrm{SC}}$. While these assignments may not be suitable for obtaining precise absolutes values of the gap magnitudes, they are likely sufficient to obtain reasonable and consistent trends concerning the doping dependence of the gap magnitudes.

Figure 4 summarizes the doping phase diagram of the obtained gap magnitudes $\Delta_{\mathrm{PG}}$ and $\Delta_{\text {SC }}$. It also shows the corresponding values that have been reported from high resolution ARPES measurements [11]. Apparently they reveal a very similar doping dependence and they even agree well concerning the absolute values. It is evident from Fig. 4 that $\Delta_{\mathrm{PG}}$ exhibits a very different and much stronger doping dependence than $\Delta_{\mathrm{SC}}$, which remains almost constant on the underdoped side, except for the very underdoped region where it suddenly decreases as shown by the ARPES data. In particular, we find that $\Delta_{\mathrm{PG}}$ is very large at strong underdoping but decreases steeply as the doping increases and even falls below $\Delta_{\mathrm{SC}}$ at $p>0.12$. Towards the overdoped side, we cannot follow the evolution of $\Delta_{\mathrm{PG}}$ but a linear extrapolation suggests that the pseudogap vanishes around a critical doping of $p_{\text {crit }} \approx 0.19-0.2$. Notably, the vanishing of the pseudogap at the same critical doping level has been previously reported based on the analysis of specific heat and nuclear magnetic resonance data $[8,27]$. We emphasize that this trend is not compatible with an interpretation of the pseudogap in terms of a precursor superconducting state where $\Delta_{\mathrm{PG}}$ and $\Delta_{\mathrm{SC}}$ should merge on the overdoped side. We also note that the finding of $\Delta_{\mathrm{PG}}>\Delta_{\mathrm{SC}}$ at $p<0.12$ suggests that the two gaps do not simply add to the spectroscopic gap, i.e., that they do not share the same electronic states. Otherwise, the gap that forms at lower temperature, $\Delta_{\mathrm{SC}}$, would have to be larger than the one at higher temperature, $\Delta_{\mathrm{PG}}$. This observation also seems to agree with the ARPES data in Ref. [11] which reveal that the gaps are separated in $k$-space.

In summary, our analysis of the temperature- and frequency dependent changes of the electronic background in the infrared $c$-axis spectra of underdoped R-123 crystals revealed that the pseudogap below $T^{*}$ leads to a depletion of the low-energy density of states that is irreversible below $T_{\mathrm{c}}$ and thus harmful to the superconducting state. Further evidence for a distinct nature of the pseudogap correlations has been obtained from the doping dependence of $\Delta_{\mathrm{PG}}$ and $\Delta_{\mathrm{SC}}$ which do not merge in the overdoped regime. Instead our data suggest that $\Delta_{\mathrm{PG}}$ vanishes close to the critical doping level of $p_{\text {crit }} \approx 0.19$ that has been previously identified $[3,8,27,63]$. 


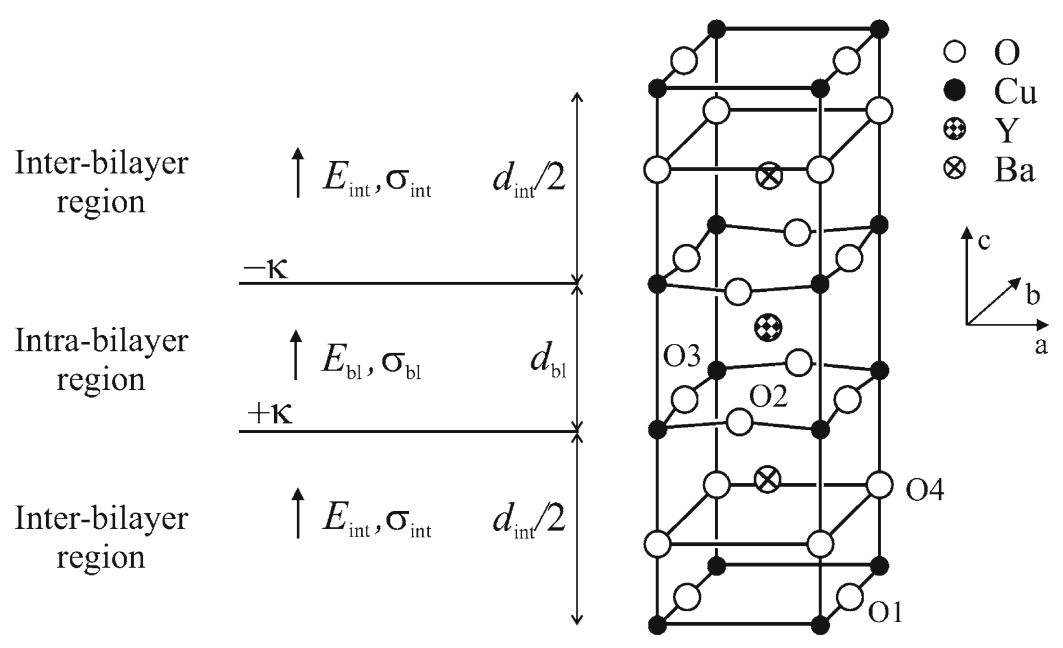

Fig. 5. Crystal structure of Y-123 with a sketch showing the intra-bilayer and the inter-bilayer regions, the local electric fields and conductivities as they are used in the multilayer model (MLM) that is described in the text.

\section{The multilayer model}

It was earlier noticed that a proper description of the $c$-axis response of the so-called bilayer compound $\mathrm{YBa}_{2} \mathrm{Cu}_{3} \mathrm{O}_{7-\delta}$, which has two $\mathrm{CuO}_{2}$ planes per unit cell, requires that one explicitly takes into account the large difference between the local conductivities between the closely spaced $\mathrm{CuO}_{2}$ planes of the bilayer unit, the so-called intra-bilayer conductivity, $\sigma_{\mathrm{bl}}$, and the ones that are more widely spaced across the $\mathrm{CuO}$ chain layer, the inter-bilayer conductivity, $\sigma_{\text {int }}$ (see Fig. 5). This is the concept of the so-called multilayer model (MLM) [43-46] which provides a surprisingly good description of the $c$-axis response as was shown for the bilayer compounds $\mathrm{YBa}_{2} \mathrm{Cu}_{3} \mathrm{O}_{7-\delta}[45,46]$ and $\mathrm{Bi}_{2} \mathrm{Sr}_{2} \mathrm{CaCu}_{2} \mathrm{O}_{8+\delta}$ [64], as well as the trilayer compound $\mathrm{Bi}_{2} \mathrm{Sr}_{2} \mathrm{Ca}_{2} \mathrm{Cu}_{3} \mathrm{O}_{10+\delta}(\mathrm{Bi}-2223)$ [47].

Due to the difference between $\sigma_{\mathrm{bl}}$ and $\sigma_{\text {int }}$ the application of an oscillatory electric field along the $c$-axis leads to charge oscillations between the $\mathrm{CuO}_{2}$ planes which are infrared active and give rise to an electronic band with a finite frequency of about 300-600 $\mathrm{cm}^{-1}$ (depending on $p$ ). This band has been termed the transverse plasma mode (TPM). This TPM gives rise to a strong modification of the local dynamical electric fields which are acting on the ions that participate in the phonon modes. Accordingly, it is responsible for the anomalous temperature dependence of some of the infrared-active $c$-axis phonon modes. It was indeed shown that the MLM provides an excellent description not only of the TPM but also of the sizeable anomalies of the $c$-axis phonon modes [46-48,64]. It turns out that the renormalization of the phonons is directly linked with the charge oscillations. In return, as shown below, the phonon anomalies thus can serve as an extremely sensitive probe for the coherency of the intra-bilayer conductivity, $\sigma_{\mathrm{bl}}$, which allows one to conveniently detect the onset of a precursor superconducting state.

Below we summarize the MLM model. A detailed derivation of the equations can be found in Ref. [48]. The $c$-axis dielectric function in the optical limit is expressed as

$$
\epsilon(\omega)=\epsilon_{\infty}+\frac{\mathrm{i}}{\epsilon_{0} \omega} \frac{\sum_{k}\left\langle j_{k}(\omega)\right\rangle}{E(\omega)}
$$

where $\epsilon_{\infty}$ is the interband dielectric constant, $\left\langle j_{\mathrm{k}}(\omega)\right\rangle$ is a volume averaged microscopic current densities and $E(\omega)$ denotes the total electric field along the $c$-axis. For a bilayer compound, 
Eq. (2) can be further rewritten as

$$
\begin{aligned}
\epsilon(\omega)= & \epsilon_{\infty}+\frac{d_{\mathrm{bl}}}{d_{\mathrm{bl}}+d_{\mathrm{int}}} \frac{\mathrm{i}}{\epsilon_{0} \omega} \frac{j_{\mathrm{bl}}(\omega)}{E(\omega)}+\frac{d_{\mathrm{int}}}{d_{\mathrm{bl}}+d_{\mathrm{int}}} \frac{\mathrm{i}}{\epsilon_{0} \omega} \frac{j_{\mathrm{int}}(\omega)}{E(\omega)}+ \\
& +\chi_{\mathrm{int}}^{Q}(\omega) \frac{E_{\mathrm{int}}^{\mathrm{loc}}(\omega)}{E(\omega)}+\chi_{\mathrm{ocp}}^{Q}(\omega) \frac{E_{\mathrm{ocp}}^{\mathrm{loc}}(\omega)}{E(\omega)}+\chi_{\mathrm{Lor}}^{Q}(\omega),
\end{aligned}
$$

where the second and third term are the contributions from the intra-bilayer and inter-bilayer current densities, respectively. These current densities are given by corresponding polarizabilities and local averaged fields,

$$
\begin{gathered}
j_{\mathrm{bl}}(\omega)=-\mathrm{i} \omega \epsilon_{0} \chi_{\mathrm{bl}}(\omega) E_{\mathrm{bl}}(\omega), \\
j_{\mathrm{int}}(\omega)=-\mathrm{i} \omega \epsilon_{0} \chi_{\mathrm{int}}(\omega) E_{\mathrm{int}}(\omega) .
\end{gathered}
$$

The local polarizabilities, $\chi_{\mathrm{bl}}(\omega)$ and $\chi_{\mathrm{int}}(\omega)$, differ and for the relevant infrared range we assume that they can be represented by Drude-Lorentz terms

$$
\begin{aligned}
& \chi_{\mathrm{bl}}(\omega)=-\frac{\omega_{\mathrm{bl}}^{2}}{\omega^{2}}-\frac{\Omega_{\mathrm{bl}}^{2}}{\omega\left(\omega+\mathrm{i} \gamma_{\mathrm{bl}}\right)}+\sum_{j} \frac{S_{\mathrm{bl}, j}^{2}}{\nu_{\mathrm{bl}, j}^{2}-\omega^{2}-\mathrm{i} \omega \Gamma_{\mathrm{bl}, j}} \text { and } \\
& \chi_{\mathrm{int}}(\omega)=-\frac{\Omega_{\mathrm{int}}^{2}}{\omega^{2}}-\frac{\omega_{\mathrm{int}}^{2}}{\omega\left(\omega+\mathrm{i} \gamma_{\mathrm{int}}\right)}+\sum_{j} \frac{S_{\mathrm{int}, j}^{2}}{\nu_{\mathrm{int}, j}^{2}-\omega^{2}-\mathrm{i} \omega \Gamma_{\mathrm{int}, j}},
\end{aligned}
$$

respectively. The first and the second term in these equations account for the response of the superconducting condensate and the quasiparticles with finite scattering rate (Drude term), respectively. The sum in Eqs. (6) and (7) represents the additional oscillators that are necessary to fit the higher energy part of the spectra above $1000 \mathrm{~cm}^{-1}$.

The last three terms of Eq. (3) represent the contribution from vibrations of ions that are (i) within the inter-bilayer area ('int'), (ii) on the copper oxygen planes ('ocp'), the outer ones in case of a trilayer, and (iii) a 'mean-field' vibration. Below, we use the 'int' vibrations to model the Y-123 phonons at 280, 560 and $620 \mathrm{~cm}^{-1}$ and the 'ocp' vibrations for the phonon at $320 \mathrm{~cm}^{-1}$. These vibrations are driven by the local fields $E_{\text {int }}^{\text {loc }}(\omega)$ and $E_{\text {ocp }}^{\text {loc }}(\omega)$, respectively. The 'mean-field' term $\chi_{\text {Lor }}^{Q}(\omega)$ in Eq. (3) corresponds to the vibrations driven by the total average field $E(\omega)$ and thus to non-interacting phonons. This term is used to account for the phonons below $220 \mathrm{~cm}^{-1}$ that either do not exhibit any pronounced phonon anomaly or only have a very small spectral weight. All the bare polarizabilities, $\chi_{\text {int }}^{Q}(\omega), \chi_{\text {ocp }}^{Q}(\omega), \chi_{\text {Lor }}^{Q}$ are expressed as the sum of Lorentzian oscillators

$$
\begin{aligned}
\chi_{\mathrm{ocp}}^{Q}(\omega) & =\sum_{k} \frac{\omega_{\mathrm{pl}, \mathrm{ocp}, k}^{2}}{\omega_{0, \mathrm{ocp}, k}^{2}-\omega^{2}-\mathrm{i} \omega g_{\mathrm{ocp}, k}}, \\
\chi_{\mathrm{int}}^{Q}(\omega) & =\sum_{k} \frac{\omega_{\mathrm{pl}, \mathrm{int}, k}^{2}}{\omega_{0, \mathrm{int}, k}^{2}-\omega^{2}-\mathrm{i} \omega g_{\mathrm{int}, k}}, \\
\chi_{\mathrm{Lor}}^{Q}(\omega) & =\sum_{k} \frac{\omega_{\mathrm{pl}, \mathrm{Lor}, k}^{2}}{\omega_{0, \mathrm{Lor}, k}^{2}-\omega^{2}-\mathrm{i} \omega g_{\mathrm{Lor}, k}} .
\end{aligned}
$$

The local electric fields are obtained with the help of following equations. The local average field of the intra-bilayer region, $E_{\mathrm{bl}}(\omega)$, is given by

$$
E_{\mathrm{bl}}(\omega)=E^{\prime}(\omega)+\frac{\kappa(\omega)}{\epsilon_{0} \epsilon_{\infty}}-\frac{d_{\mathrm{int}}+d_{\mathrm{bl}}}{\epsilon_{\infty} d_{\mathrm{bl}}} \chi_{\mathrm{bl}}^{Q}(\omega) E_{\mathrm{bl}}^{\mathrm{loc}}(\omega)-\frac{d_{\mathrm{int}}+d_{\mathrm{bl}}}{2 \epsilon_{\infty} d_{\mathrm{bl}}} \chi_{\mathrm{ocp}}^{Q}(\omega) E_{\mathrm{ocp}}^{\mathrm{loc}}(\omega) .
$$


and the local average electric field of the inter-bilayer region, $E_{\text {int }}(\omega)$, is given by

$$
E_{\mathrm{int}}(\omega)=E^{\prime}(\omega)-\frac{d_{\mathrm{int}}+d_{\mathrm{bl}}}{\epsilon_{\infty} d_{\mathrm{int}}} \chi_{\mathrm{int}}^{Q}(\omega) E_{\mathrm{int}}^{\mathrm{loc}}(\omega)-\frac{d_{\mathrm{int}}+d_{\mathrm{bl}}}{2 \epsilon_{\infty} d_{\mathrm{bl}}} \chi_{\mathrm{ocp}}^{Q}(\omega) E_{\mathrm{ocp}}^{\mathrm{loc}}(\omega) .
$$

The total average electric field of the whole elementary cell, $E(\omega)$, is given by the average of the two latter fields

$$
E(\omega)=\frac{d_{\mathrm{bl}}}{d_{\mathrm{bl}}+d_{\mathrm{int}}} E_{\mathrm{bl}}(\omega)+\frac{d_{\mathrm{int}}}{d_{\mathrm{bl}}+d_{\mathrm{int}}} E_{\mathrm{int}}(\omega) .
$$

$E^{\prime}(\omega)$ is the component of the total average internal field $E(\omega)$ that is due to external charges and depolarization processes at high frequencies.

The local electric fields acting on the ions reads

$$
\begin{gathered}
E_{\text {int }}^{\text {loc }}(\omega)=E^{\prime}(\omega), \\
E_{\text {ocp }}^{\text {loc }}(\omega)=E^{\prime}(\omega)+\frac{\kappa(\omega)}{2 \epsilon_{0} \epsilon_{\infty}} .
\end{gathered}
$$

For completeness, we specify the local electric field acting on an ion within the intra-bilayer region

$$
E_{\mathrm{bl}}^{\mathrm{loc}}(\omega)=E^{\prime}(\omega)+\frac{\kappa(\omega)}{\epsilon_{0} \epsilon_{\infty}} .
$$

The conservation of charge reads

$$
j_{\mathrm{bl}}(\omega)-j_{\mathrm{int}}(\omega)=\mathrm{i} \omega \kappa(\omega) .
$$

Equations (3)-(17) represent a set of linear algebraic equations which describe self-consistently the interacting system of the electric field, the electric current densities and the vibrating charged ions. Since the conductivity varies substantially on the nanometer scale along the $c$-axis direction, the local electric fields can exhibit large variations, e.g. they can even change their sign within the unit cell [47].

\section{Evidence for precursor superconductivity in underdoped $\mathrm{YBa}_{2} \mathrm{Cu}_{3} \mathrm{O}_{6.6}$}

In this paragraph we describe how the local conductivities

$$
\begin{gathered}
\sigma_{\mathrm{bl}}(\omega)=-\mathrm{i} \omega \epsilon_{0} \chi_{\mathrm{bl}}(\omega), \\
\sigma_{\mathrm{int}}(\omega)=-\mathrm{i} \omega \epsilon_{0} \chi_{\mathrm{int}}(\omega)
\end{gathered}
$$

are obtained from fits with the multilayer mode (MLM) of the experimental spectra of the $c$-axis conductivity of an underdoped $\mathrm{YBa}_{2} \mathrm{Cu}_{3} \mathrm{O}_{6.6}$ crystal with $T_{\mathrm{c}}=58 \mathrm{~K}$. We also show that the obtained very different frequency- and temperature dependence of $\sigma_{\mathrm{bl}}$ and $\sigma_{\mathrm{int}}$ enables one to disentangle the signatures of the extrinsic pseudogap below $T^{*}$ from the one of the precursor superconducting state that occurs below $T^{\mathrm{ons}}<T^{*}$.

Figure 6(a) and 6(b) compare the measured spectra of $\sigma_{1 c}(\omega)$ and $\sigma_{2 c}(\omega)$ (symbols) with the fits (solid lines) and they show that the MLM provides a very good description of the experimental data. In particular, the MLM reproduces the characteristic features, such as (i) the TPM near $450 \mathrm{~cm}^{-1}$, (ii) the related anomaly of the $320 \mathrm{~cm}^{-1}$ phonon mode, and (iii) the gradual suppression of the broad electronic continuum due to the extrinsic pseudogap [22-25]. As previously described in Ref. $[29,58]$, the latter gives rise to an upward shift of the low-energy spectral weight into a band just above the gap edge around $2 \Delta_{\mathrm{PG}} \approx 1200 \mathrm{~cm}^{-1}$.

Before discussing the results in more detail, we note the following assumptions that we made in fitting the data. In order to reduce the number of free parameter, we assumed a temperature 


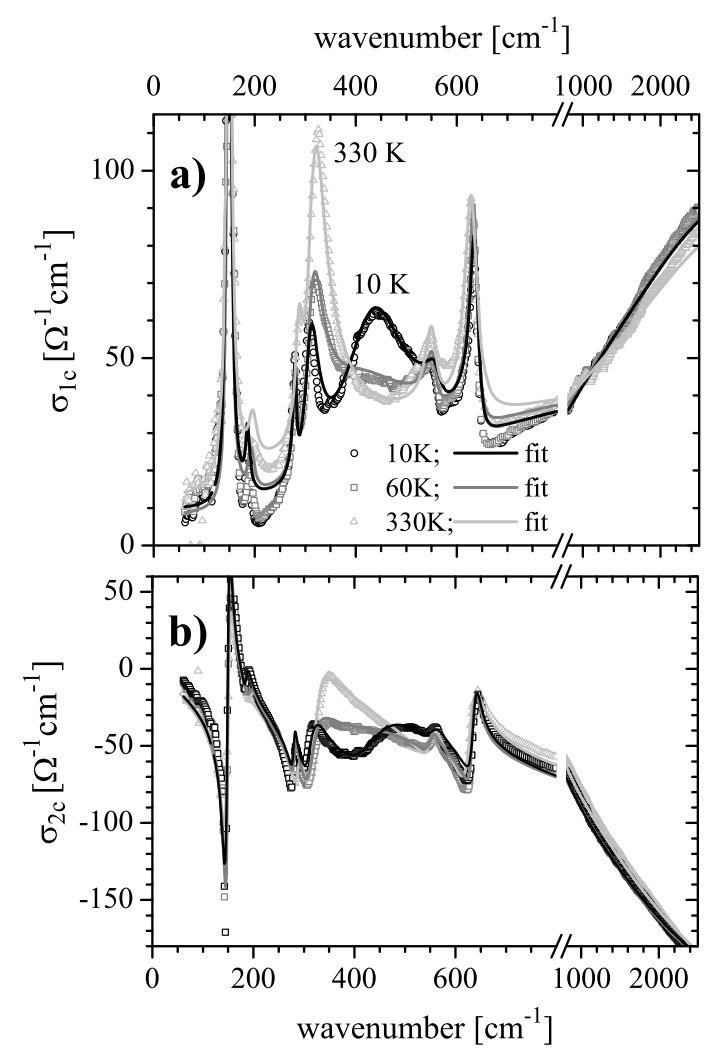

Fig. 6. Comparison of the measured spectra (symbols) and the fits with the MLM (solid lines) of (a) the real part and (b) the imaginary part of the $c$-axis conductivity of underdoped $\mathrm{YBa}_{2} \mathrm{Cu}_{3} \mathrm{O}_{6.6}$ with $T_{\mathrm{c}}=58 \mathrm{~K}$ (from Ref. [65]).

independent broadening of the Drude term of $\gamma_{\mathrm{bl}}=150 \mathrm{~cm}^{-1}$. This assumption is motivated by our finding that $\gamma_{\mathrm{bl}}$ does not change very much at $T>T_{\mathrm{c}}$ if it is treated as a free parameter (see below). The increased coherency of the low-frequency response at $T<T_{\mathrm{c}}$ has been entirely accounted for in terms of the delta-function like response which represents the loss-free response due to the macroscopically coherent superconducting condensate. Below we show that this does not affect the temperature dependence of the coherent spectral weight in $\sigma_{\mathrm{bl}}$ since qualitatively similar result is obtained if we only use a Drude-response with a variable broadening parameter, $\gamma_{\mathrm{bl}}$. For the fitting, we have used both the real and imaginary part of the conductivity function that have been determined independently by ellipsometry.

Furthermore, in order to reproduce the high energy part above $1000 \mathrm{~cm}^{-1}$ which is only weakly temperature dependent, we used two broad oscillators located at about 1500 and $3000 \mathrm{~cm}^{-1}$. As previously outlined [46], the phonons at 280,560 and $620 \mathrm{~cm}^{-1}$ are predominantly due to the vibration of oxygens in the inter-bilayer region [term $\chi_{\text {int }}^{Q}(\omega)$ in Eq. (3)] and the $320 \mathrm{~cm}^{-1}$ phonon is due to the bond-bending vibration of oxygens in $\mathrm{CuO}_{2}$ planes [term $\chi_{\text {ocp }}^{Q}(\omega)$ in Eq. (3)]. Finally, the contributions of the phonons below $220 \mathrm{~cm}^{-1}$ has been modelled with standard Lorentzian oscillators [term $\chi_{\text {Lor }}^{Q}(\omega)$ in Eq. (3)]. A very important role in our analysis is played by the $320 \mathrm{~cm}^{-1}$ phonon mode. We stress that its bare oscillator strength and bare frequency were kept fixed for all temperatures at $\omega_{\mathrm{pl}, \mathrm{ocp}}=481 \mathrm{~cm}^{-1}$ and $\omega_{0, \text { ocp }}=410 \mathrm{~cm}^{-1}$. Therefore all the large changes of the oscillator strength and the frequency of the phonon lineshape are due to the coupling to the inter- and intra-bilayer currents and their corresponding changes with temperature.

The spectra of $\sigma_{1, \text { int }}$ and $\sigma_{1, \mathrm{bl}}$, as obtained with the MLM, are detailed in Figs. 7(a) and 7(b). They differ remarkably concerning their absolute values as well as their temperature- and 

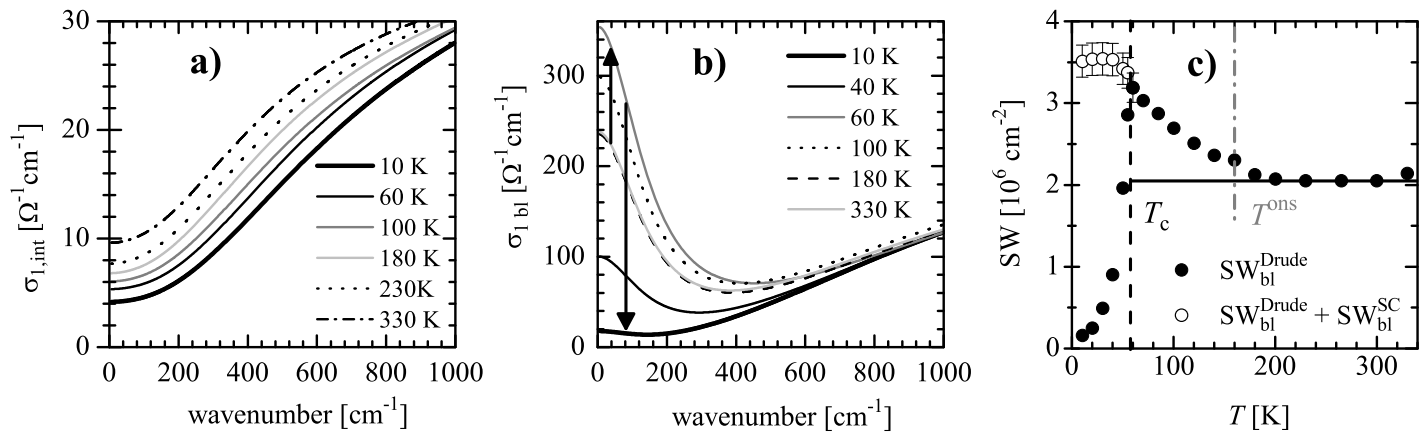

Fig. 7. Spectra of (a) the inter-bilayer conductivity, $\sigma_{1, \text { int }}(\omega)$, and (b) the intra-bilayer conductivity, $\sigma_{1, \mathrm{bl}}(\omega)$, as obtained by fitting the experimental $c$-axis conductivity of underdoped $\mathrm{YBa}_{2} \mathrm{Cu}_{3} \mathrm{O}_{6.6}$ with $T_{\mathrm{c}}=58 \mathrm{~K}$ (see Fig. 6) with the MLM model (from Ref. [65]). (c) Temperature dependence of the spectral weight of the intra-bilayer Drude response $\mathrm{SW}_{\mathrm{bl}}^{\text {Drude }}$ with finite scattering rate (solid circles) and the total coherent spectral weight $\mathrm{SW}_{\mathrm{bl}}^{\mathrm{Drude}}+\mathrm{SW}_{\mathrm{bl}}^{\mathrm{SC}}$ (open circles). $\mathrm{SW}_{\mathrm{bl}}^{\mathrm{SC}}$ is the spectral weight of the intra-bilayer $\mathrm{SC}$ condensate that emerges at $T<T_{\mathrm{c}}$.

frequency dependence. Figure 7(a) shows that the low absolute value, the spectral shape, and the temperature dependence (its decrease with decreasing temperature) of $\sigma_{1 \text {,int }}$ are characteristic of an incoherent transport. Similar to the electronic background in $\sigma_{1 \mathrm{c}}$, the evolution of $\sigma_{1 \text {,int }}$ thus appears to be determined by the extrinsic pseudogap which develops below $T^{*} \approx 300 \mathrm{~K}[29$ ] On the other hand, the spectra of $\sigma_{1, \mathrm{bl}}$ in Fig. $7(\mathrm{~b})$ reveal a clear Drude-like response. We note that the main temperature dependent changes of the intra-bilayer current occur below $700 \mathrm{~cm}^{-1}$ and account for the TPM and the related phonon anomalies in the spectra of $\sigma_{1 \mathrm{c}}$. In contrast, the corresponding changes of inter-bilayer response are connected with the very slowly varying electronic background and the related spectral weight shift from low to high energies above $1000 \mathrm{~cm}^{-1}$ [29]. Therefore, the two currents are responsible for clearly distinct features in shape and frequency and thus their determination is well conditioned.

The temperature dependence of the intra-bilayer response is detailed in the Fig. 7 (c) in terms of the spectral weight of the coherent low-frequency response. Closed circles represent the spectral weight of the response with a finite scattering rate, $\mathrm{SW}_{\mathrm{bl}}^{\text {Drude }}=\Omega_{\mathrm{bl}}^{2}$. Open symbols represent the sum of $\mathrm{SW}_{\mathrm{bl}}^{\mathrm{Drude}}$ and the spectral weight of the delta function at $\omega=0$, $\mathrm{SW}_{\mathrm{bl}}^{\mathrm{SC}}=\omega_{\mathrm{bl}}^{2}$, that emerges below $T_{\mathrm{c}}$ and represents the loss-free response of the macroscopically coherent superconducting condensate. The latter has been obtained from the spectra of $\sigma_{2, \mathrm{bl}}$. This coherent spectral weight hardly changes between 330 and $180 \mathrm{~K}$ while it starts to exhibit a significant increase below $T^{\text {ons }} \approx 160 \mathrm{~K}$. It is also evident that at $T<T_{\mathrm{c}}$, the vast majority of the low-frequency spectral weight eventually is redistributed from the Drude-like band to the delta function. We emphasize that the scenario of a precursor SC state naturally accounts for these trends in $\sigma_{\mathrm{bl}}$. The increase of the coherent spectral weight below $T^{\text {ons }}$ is explained in terms of the precursor $\mathrm{SC}$ gap which, similar to the $\mathrm{SC}$ gap below $T_{\mathrm{c}}$, gives rise to a redistribution of SW to low frequencies. The finite width of the Drude-like band at $T_{\mathrm{c}}<T<T^{\mathrm{ons}}$ is due to the finite correlation time in the fluctuating precursor superconducting state. Finally, as the fluctuations diminish below $T_{\mathrm{c}}$, almost the entire $\mathrm{SW}_{\mathrm{bl}}^{\text {Drude }}$ is transferred to the delta-function that represents the loss-free response of the macroscopically coherent condensate.

In the following we show that a qualitatively similar result concerning the increase of the coherent low-frequency spectral weight in $\sigma_{\text {bl }}$ below $T^{\text {ons }}$ is obtained even if we do not fix the value of $\gamma_{\mathrm{bl}}$. In this case, the Drude term gets very narrow at $T \ll T_{\mathrm{c}}\left(\right.$ with $\gamma_{\mathrm{bl}}<10 \mathrm{~cm}^{-1}$ ) and we do not need to include an additional delta function at $\omega=0$ below $T_{\mathrm{c}}$. All the spectral weight of the coherent low frequency response is thus contained in the Drude term, i.e. in $\mathrm{SW}_{\mathrm{bl}}^{\text {Drude }}=\Omega_{\mathrm{bl}}^{2}$. Figure 8(a) shows that this different parameterization of $\sigma_{\mathrm{bl}}$ also allows for a good description of the experimental data and Fig. 8(b) and 8(c) confirm that the obtained trends are similar as in the case where $\gamma_{\mathrm{bl}}$ was fixed. In particular, SW $\mathrm{Sb}$ Drude is essentially 

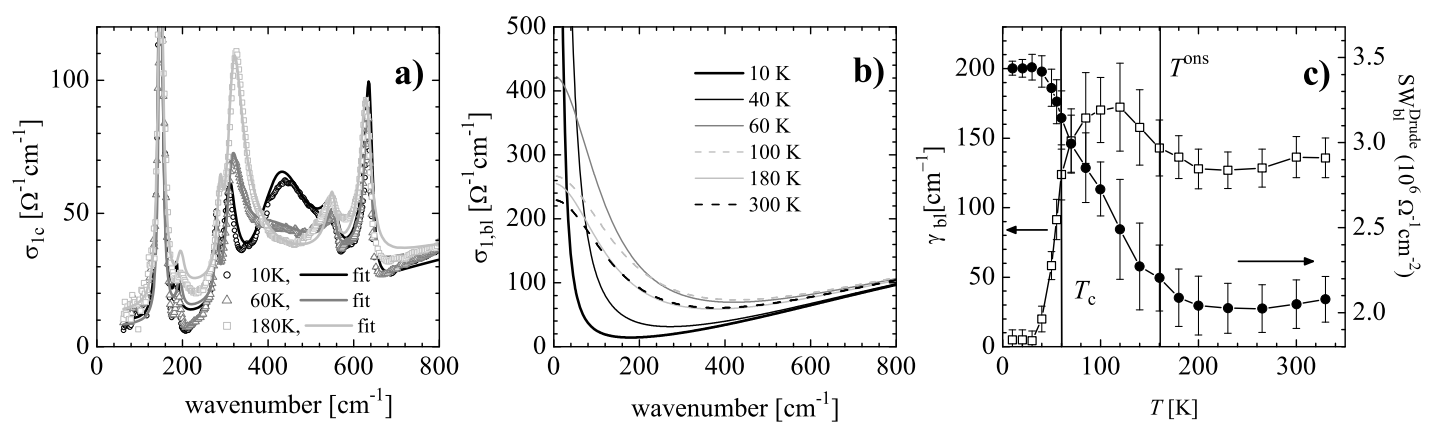

Fig. 8. (a) Comparison of the measured spectra of the $c$-axis conductivity of underdoped $\mathrm{YBa}_{2} \mathrm{Cu}_{3} \mathrm{O}_{6.6}$ with $T_{\mathrm{c}}=58 \mathrm{~K}$ and the fits with the MLM where the broadening of the Drude term in $\sigma_{\mathrm{bl}}$ has not been fixed. (b) The obtained spectra of the real part of the intra-bilayer conductivity, $\sigma_{1, \mathrm{bl}}$. (c) Temperature dependence of the spectral weight, $\mathrm{SW}_{\mathrm{bl}}^{\text {Drude }}$, and the broadening, $\gamma_{\mathrm{bl}}$, of the Drude term.

constant above $T^{\text {ons }} \approx 160 \mathrm{~K}$, while it exhibits a clear increase below $T^{\text {ons }}$ that is very similar in magnitude as for the case of fixed $\gamma_{\mathrm{bl}}$ [see Fig. $7(\mathrm{c})$ ]. The value of $\gamma_{\mathrm{bl}}$ can be seen to exhibit only a small variation above $T_{\mathrm{c}}$. As such it can be concluded that the model with a fixed width of the Drude term, as presented in Fig. 6 and 7, provides a reasonable estimate for the increase of the coherent spectral weight below $T^{\text {ons }}$. Finally, below $T_{\mathrm{c}}$ the value of $\gamma_{\mathrm{bl}}$ decreases sharply and almost vanishes at $T \ll T_{\mathrm{c}}$.

We note that some of the systematic errors as seen in Figs. 6(a) and 8(a) may arise from the assumptions made in the MLM concerning the eigenvectors of the phonons above $200 \mathrm{~cm}^{-1}$ (particularly the one at $320 \mathrm{~cm}^{-1}$ ). For the sake of simplicity, we only take into account the dominant contributions of the shell model based polarization diagrams [68]. In particular, we use simplified polarization diagrams where only the ions of the $\mathrm{CuO}_{2}$ planes, intra- or interbilayer area are vibrating. While the fits could be improved by allowing several type of ions to vibrate, as in Ref. $[47,48]$, this would mostly increase the complexity of the model but hardly yield more accurate results for the local conductivity, $\sigma_{\mathrm{bl}}$, which is the main subject of the present work.

Finally, this leaves us with the question why the local inter-bilayer and intra-bilayer conductivities, $\sigma_{\mathrm{int}}$ and $\sigma_{\mathrm{bl}}$, exhibit such fundamental differences in their frequency and temperature such that one of them is most sensitive to the competing pseudogap that depletes the density of low-energy states that are available for superconductivity, while the other reveals the onset of a precursor superconducting state below $T^{\text {ons }}$. This can be understood as follows:

In bilayer cuprate superconductors like Y-123, the conduction band of the copper-oxygen planes is split into bonding band (B) and antibonding band (A), respectively. Their dispersions are conveniently parameterized as

$$
\epsilon_{B / A}\left(\mathbf{k}_{\|}, k_{z}\right)=\epsilon\left(\mathbf{k}_{\|}\right) \mp \sqrt{t_{\perp \text { bl }}^{2}\left(\mathbf{k}_{\|}\right)+t_{\perp \text { int }}^{2}\left(\mathbf{k}_{\|}\right)+2 t_{\perp \text { bl }}\left(\mathbf{k}_{\|}\right) t_{\perp \text { int }}\left(\mathbf{k}_{\|}\right) \cos \left(k_{z} d\right)} .
$$

Here $\epsilon$ is the in-plane component, that can be expressed in terms of hopping matrix elements between orbitals of a single plane. The parameters $t_{\perp \text { bl }}$ and $t_{\perp \text { int }}$ are the $\mathbf{k}_{\|}$-dependent hopping matrix elements between the closely-spaced planes and the widely-spaced planes, respectively, that can be expressed in terms of hopping matrix elements between orbitals of different planes. The magnitude of $t_{\perp}$ bl is much higher than that of $t_{\perp \text { int }}$. It follows from Eq. (20) that the splitting of the conduction band is approximately equal to $2 t_{\perp} \mathrm{bl}$ :

$$
\Delta \epsilon=\epsilon_{A}-\epsilon_{B} \approx 2 t_{\perp \mathrm{bl}} .
$$

According to the analysis by O. Andersen and coworkers [69], the inter-plane hopping proceeds mainly through the copper 4 s orbitals. Considerations of this hopping channel yield [69]

$$
t_{\perp \mathrm{int} / \mathrm{bl}} \approx \frac{t_{\perp \mathrm{int} / \mathrm{bl}}(X)}{4}\left[\cos \left(k_{x} a\right)-\cos \left(k_{y} a\right)\right]^{2},
$$


where $X$ stands for $(\pi / a, 0)$ and $a$ is the lattice parameter. The inter-bilayer hopping in Y123 involves the apical oxygen $2 p_{z}$ orbitals, that can be accessed from the copper $4 \mathrm{~s}$ only. This suggests that Eq. (22) provides a realistic approximation to $t_{\perp \text { int }}$. The hopping thus can be expected to attain its maximum at the $X$-point (in the so-called antinodal region where the extrinsic pseudogap prevails) to vanish along the BZ diagonal and to be small around the diagonal, in the so called nodal region, where well-defined quasiparticles persist even in strongly underdoped samples. The calculated $\mathbf{k}_{\|}$-dependence of $t_{\perp}$ bl (see Fig. 7 (b) of Ref. [69]) exhibits a maximum close to the $X$-point, consistent with the considerations of the copper $4 \mathrm{~s}$ channel, but the magnitude of $t_{\perp \text { bl }}$ at the BZ diagonal is only by a factor of about 2 smaller than that at the $X$-point. Photoemission experiments on Y-123 [71,72] suggest an even weaker anisotropy of the effective intra-bilayer hopping which, consistent with Eq. (21), is defined as one half of the bilayer splitting. It is thus the strong $k$-space asymmetry of the inter-planar hopping matrix elements which is most likely responsible for these pronounced differences between, $\sigma_{\text {int }}$ and $\sigma_{\mathrm{bl}}$. In addition, it should be noted that the magnitude of $t_{\perp \text { int }}$ is much smaller than the one of $t_{\perp \text { bl }}$ and likely even considerably smaller than the in-plane scattering rate at $T>T_{\mathrm{c}}$ such that a small contribution from the quasiparticles of the near-nodal region would not show up as a coherent response in $\sigma_{\text {int }}$.

\section{Summary}

We applied the technique of infrared ellipsometry to investigate in detail the doping-, temperature- and frequency dependence of the $c$-axis response of a series of weakly overdoped to strongly underdoped $\mathrm{RBa}_{2} \mathrm{Cu}_{3} \mathrm{O}_{7-\delta}(\mathrm{R}=\mathrm{Y}$ and $\mathrm{Nd})$ single crystals. Our emphasis has been on the anomalous electronic properties at temperatures above the macroscopic superconducting transition temperature, $T_{\mathrm{c}}$, and the question whether they are caused by a precursor superconducting state or rather by electronic correlations that compete with superconductivity. We have found that the $c$-axis spectra provide evidence that both kinds of correlations are present and that their contributions can be disentangled based on an analysis with the so-called multilayermodel. The evidence of the competing pseudogap can be obtained from the as measurements spectra. We showed that the onset temperature, $T^{*}$, and the energy scale, $\Delta_{\mathrm{PG}}$, of this competing pseudogap increase rapidly towards the underdoped side whereas they vanish on the overdoped side. On the other hand, we found that the signatures of the precursor superconducting state are contained in the local conductivity between the closely spaced $\mathrm{CuO}_{2}$ planes of the so-called bilayer unit. We showed how this local response can be obtained with the multilayer model and for a strongly underdoped sample we showed that the onset temperature of precursor superconductivity, $T^{\mathrm{ons}}$, is considerably lower than $T^{*}$ but still much higher than $T_{\mathrm{c}}$.

Part of the work was performed at the IR-beamline of the ANKA synchrotron at FZ Karlsruhe, D, where we acknowledge support of Y.L. Mathis. The work was financially supported by the Deutsche Forschungsgemeinschaft (DFG) with grant BE2684/1 in FOR538, by the Schweizerischer Nationalfonds (SNF) with grants 200020-119784, 200020-129484, and the NCCR-MaNEP, as well as the Ministry of Education of the CR (MSM002162401).

\section{References}

1. J.W. Loram, K.A. Mirza, J.R. Cooper, W.Y. Liang, J.M. Wade, J. Supercon. 7, 243 (1994)

2. T. Timusk, B. Statt, Rep. Prog. Phys. 62, 61 (1999)

3. J.L. Tallon, et al., Phys. Stat. Sol. (b) 215, 531 (1999)

4. J.W. Loram, J. Luo, J.R. Cooper, W.Y. Liang, J.L. Tallon, J. Phys. Chem. Solids 62, 59 (2001)

5. M.R. Presland, J.L. Tallon, R.G. Buckley, R.S. Liu, N.E. Flower, Physica C 176, 95 (1991)

6. J.L. Tallon, C. Bernhard, H. Shaked, R.L. Hitterman, J.D. Jorgensen, Phys. Rev. B 51, 12911 (1995)

7. H. Alloul, T. Ohno, P. Mendels, Phys. Rev. Lett. 63, 1700 (1989) 
8. J.W. Loram, K.A. Mirza, J.R. Cooper, W.Y. Liang, Phys. Rev. Lett. 71, 1740 (1993)

9. A.G. Loeser, et al., Science 273, 325 (1996)

10. H. Ding, et al., Nature 382, 51 (1996)

11. K. Tanaka, et al., Science 314, 1910 (2006)

12. V.M. Krasnov, A. Yurgens, D. Winkler, P. Delsing, T. Claeson, Phys. Rev. Lett. 84, 5860 (2000)

13. M. Kugler, O. Fischer, C. Renner, S. Ono, Y. Ando, Phys. Rev. Lett. 86, 4911 (2001)

14. N. Miyakawa, et al., Physica C 364, 475 (2001)

15. O. Fischer, M. Kugler, I. Maggio-Aprile, C. Berthod, C. Renner, Rev. Mod. Phys. 79, 353 (2007)

16. K.K. Gomes, et al., Nature 447, 569 (2007)

17. J.W. Alldredge, et al., Nature Physics 4, 319 (2008)

18. K. Chatterjee, et al., Nature Physics 4, 108 (2008)

19. G. Deutscher, Nature 397, 410 (1999)

20. Z.A. Xu, et al., Nature 406, 486 (2000)

21. C. Meingast, et al., Phys. Rev. Lett. 86, 1606 (2001)

22. C.C. Homes, T. Timusk, R. Liang, D.A. Bonn, W.N. Hardy, Phys. Rev. Lett. 71, 1645 (1993)

23. J. Schützmann, et al., Phys. Rev. B 52, 13665, (1995)

24. C. Bernhard, et al., Phys. Rev. Lett. 80, 1762 (1998)

25. C. Bernhard, et al., Phys. Rev. B 59, R6631 (1999)

26. D.N. Basov, T. Timusk, Rev. Mod. Phys. 77, 721 (2005)

27. J.L. Tallon, J.W. Loram, Physica C 349, 53 (2001)

28. C. Bernhard, et al., Phys. Rev. B 62, 9138 (2000)

29. Li Yu, et al., Phys. Rev. Lett. 98, 177004 (2008)

30. V.J. Emery, S.A. Kivelson, Nature 374, 434 (1995)

31. I. Kosztin, Q.J. Chen, Y.J. Kao, K. Levin, Phys. Rev. B 61, 11662 (2000)

32. P.W. Anderson, et al., J. Phys. Condensed Mat. 16, R755 (2004)

33. T. Dahm, D. Manske, L. Tewordt, Phys. Rev. B 56, 11419 (1997)

34. A.V. Chubukov, J. Schmalian, Phys. Rev. B 57, R11085 (1998)

35. C.M. Varma, Phys. Rev. Lett. 83, 3538 (1999)

36. C.C. Homes, T. Timusk, D.A. Bonn, R. Liang, W.N. Hardy, Physica C 254, 265 (1995)

37. C.C. Homes, T. Timusk, D.A. Bonn, R. Liang, W.N. Hardy, Can. J. Phys. 73, 663 (1995)

38. C. Bernhard, et al., J. Phys. Chem. Solids 69, 3064 (2008)

39. M. Tinkham, Introduction to Superconductivity (McGraw-Hill, Singapore, 1996)

40. Y. Wang, L. Li, N.P. Ong, Phys. Rev. B 73, 024510 (2006)

41. L. Li, et al., Phys. Rev. B 81, 054510 (2010)

42. J.L. Tallon, J.G. Storey, J.W. Loram (2009) [arXiv0908.4428v1]

43. C. Bernhard, et al., Phys. Rev. B 61, 618 (2000)

44. D. van der Marel, A. Tsvetkov, Czech. J. Phys. 46, 3165 (1996)

45. M. Grüninger, D. van der Marel, A.A. Tsvetkov, A. Erb, Phys. Rev. Lett. 84, 1575 (2000)

46. D. Munzar, C. Bernhard, A. Golnik, J. Humlíček, M. Cardona, Sol. Stat. Comm. 112, 365 (1999)

47. A.V. Boris, et al., Phys. Rev. Lett. 89, 277001 (2002)

48. A. Dubroka, D. Munzar, Physica C 405, 133 (2004)

49. C.T. Lin, W. Zhou, W.Y. Liang, E. Schonherr, H. Bender, Physica C 195, 291 (1992)

50. S.I. Schlachter, et al., Int. J. Modern Phys. B 14, 3673 (2000)

51. C. Bernhard, J.L. Tallon, Phys. Rev. B 54, 10201 (1996)

52. J.L. Tallon, et al., Phys. Rev. B 53, 11972 (1996)

53. S.D. Obertelli, J.R. Cooper, J.L. Tallon, Phys. Rev. B 46, 14928 (1992)

54. R. Henn, C. Bernhard, A. Wittlin, M. Cardona, S. Uchida, Thin Solid Films 313, 642 (1998)

55. A. Golnik, C. Bernhard, J. Humlíček, M. Kläser, M. Cardona, Phys. Stat. Sol. (b) 215, 553 (1999)

56. C. Bernhard, J. Humlíček, B. Keimer, Thin Solid Films 455, 143 (2004)

57. R.M.A. Azzam, N.H. Bashara, Ellipsometry and Polarized Light (North-Holland, Amsterdam, 1977)

58. A.V. Pimenov, et al., Phys. Rev. Lett. 94, 227003 (2005)

59. M. Dressel, G. Grüner, Electrodynamics of Solids (Cambridge University Press, Cambridge, 2002)

60. M.R. Norman, M. Randeira, H. Ding, J.C. Campuzano, Phys. Rev. B 57, 11093 (1998)

61. D. Munzar, J. Phys. Chem. Solids 67, 308 (2006)

62. J. Chaloupka, C. Bernhard, D. Munzar, Phys. Rev. B 79, 185413 (2009) 
63. C. Bernhard, et al., Phys. Rev. Lett. 86, 1614 (2001)

64. V. Železný, et al., Phys. Rev. B 63, 060502 (2001)

65. A. Dubroka, et al. (unpublished)

66. T. Timusk, C.C. Homes, Sol. Stat Commun. 126, 63 (2003)

67. A.D. LaForge, et al., Phys. Rev. B 76, 054524 (2007)

68. R. Henn, et al., Phys. Rev. B 55, 3285 (1997)

69. O.K. Andersen, et al., J. Phys. Chem. Solids 56, 1573 (1995)

70. T. Xiang, J.M. Wheatley, Phys. Rev. Lett. 77, 4632 (1996)

71. S.V. Borisenko, et al., Phys. Rev. Lett. 96, 117004 (2006)

72. V.B. Zabolotnyy, et al., Phys. Rev. B 76, 064519 (2007) 